\title{
Complejidad en clave docente [T0]
}

\author{
Juan David Luján Villar*
}

\section{Resumen [T1]}

Existen diferentes perspectivas de las ciencias de la complejidad (CC) y lo complejo. Para gran parte de la comunidad académica de las ciencias sociales la complejidad es sinónimo de incertidumbre (Wallerstein, 2005), para otros pensadores afines a una perspectiva literaria la complejidad constituye una forma de pensamiento (Morin, 2005). Otras miradas consideran las $\mathrm{CC}$ como un terreno que origina problemas derivados del estudio de lo social con una innumerable gama de impactos (Reynoso, 2016).

Este escrito parte de la reflexión llevada a cabo durante el desarrollo de una investigación en ciencias sociales (Luján, 2016) que estableció la complejidad como una opción en términos de problemas al intentar articular algunas de sus ideas de manera transdisciplinar, pero que quedó con una deuda epistemológica en el sentido de explicar la tarea de comprender los aspectos que encierra la complejidad más allá de la jerga habitual gestada en torno de los conceptos de complejidad no-linealidad, auto-organización, fractales y redes complejas, entre otros.

\section{Introducción [T1]}

El establecimiento de los diálogos entre las denominadas ciencias duras, las humanidades y las ciencias sociales desde una perspectiva compleja presenta el nombre de Gregory Bateson, antropólogo, epistemólogo y psiquiatra como figura pionera de primer orden.

\footnotetext{
${ }^{*}$ Magister en Investigación Social Interdisciplinaria. Secretaría de Educación Distrital, Bogotá. Integrante del grupo de investigación Literatura, Escritura y Comunicación (LEC) de la Universidad Distrital Francisco José de Caldas.
} 
[Author pre-print: Accepted versión] Citation: Luján, Juan David (2019). Complejidad en clave docente. En Sánchez, Amaya, Tomás y Durango Lara, Ibeth (comps.), Encuentro de socialización de experiencias investigativas en la Facultad de Ciencias y Educación. Memorias, número 5 (pp. 267-279). Bogotá: Universidad Distrital Francisco José de Caldas.

Desde los desarrollos de la cibernética (y el rol que desempeñó durante las Conferencias Macy de 1946 a 1953) y su teoría del doble vínculo (double-bind) se construyeron aportes invaluables a la terapia sistémica, esto último desarrollado durante su estadía en el Hospital de Veteranos de Palo Alto (1954-1959). Su influencia en el Frame Analysis de Erving Goffman y los adelantos en la teoría de la comunicación no-verbal y el estudio de las emociones de Paul Ekman —a quien suministró el material cinematográfico realizado por él durante su trabajo en Bali en 1936 - junto con Margaret Mead, hizo que muchos lo consideraran un pionero en el campo de la antropología visual.

Esta introducción atiende a las ideas que se consignarán como producto de las lecturas de Bateson, especialmente de su última obra finalizada, Espíritu y naturaleza ([1979] 1993) ${ }^{1}$. En el desarrollo de este escrito se podrá advertir cómo las ideas de este autor van y vienen respecto de la noción de aprendizaje, los problemas, las CC y el planteamiento de las recientemente definidas humanidades digitales, como un presunto aporte del vínculo entre las CC y las humanidades dentro de un posible campo transdisciplinar de trabajo investigativo, epistemológico y de comunicación de conocimiento transversalizado o no tradicional.

\section{Una idea sobre el aprendizaje [T1]}

En 1942, como comentario a un trabajo de Mead, Bateson argumentó sobre la existencia de un protoaprendizaje y un deuteroaprendizaje. Por más ruidosos que ambos términos suenen, estas ideas son bastante sencillas e igualmente profundas. En el primer caso, el aprendizaje

\footnotetext{
${ }^{1}$ Al menos dos libros recogen parte de la obra de Bateson: Lipset (1980), quien fuera discípulo de Bateson, realizó una interesante biografía en compañía de su maestro. Entretanto, el texto de Charlton (2008) intenta recoger el genio de Bateson a través de una lectura crítica de sus ideas, influencias, campos de trabajo y los aportes más relevantes de su obra.
} 
[Author pre-print: Accepted versión] Citation: Luján, Juan David (2019). Complejidad en clave docente. En Sánchez, Amaya, Tomás y Durango Lara, Ibeth (comps.), Encuentro de socialización de experiencias investigativas en la Facultad de Ciencias y Educación. Memorias, número 5 (pp. 267-279). Bogotá: Universidad Distrital Francisco José de Caldas.

de tipo memorístico es un protoaprendizaje, el cual se puede considerar como básico. A medida que el sujeto realiza una serie de acciones o secuencias cibernéticas el nivel de conocimiento se incrementará: "Este cambio progresivo en la tasa de protoaprendizaje lo llamaremos 'deuteroaprendizaje"” (Bateson, [1971] 1991, p. 195).

Según Bateson ([1971] 1991), el tránsito que se efectúa desde el protoaprendizaje hasta llegar al deuteroaprendizaje, constituye el incremento de conocimiento mediante prueba y error, respuestas acertadas y la experimentación seriada o sucesiva en el marco de un pensamiento creativo, susceptible de ser representado mediante una tasa del gradiente de deuteroaprendizaje adquirido, compuesto a través de la curva que lo grafica.

El hábito, la experiencia de la corriente de acontecimientos y el contexto se interrelacionan en la experiencia del aprendizaje. Este aprender a prender hace parte no solo de la experiencia repetida, tampoco es un único modo de aprender en la epistemología batesoniana. Los hábitos hacen referencia a la apercepción, los actos internos de reconocimiento del objeto percibido; de ahí que el autor hable de hábitos aperceptivos, esto significa de modo genérico las teorías del aprendizaje (Bateson, [1971] 1991, pp. 197$198)^{2}$

Es necesario decir que la corriente de los sucesos está mediada por lo tecnológico, lo estético, el lenguaje y los patrones culturales contrastantes. Lo anterior sobre todo se relaciona con la propuesta de deuteroaprendizaje en la medida en que la importancia de los

\footnotetext{
${ }^{2}$ Bateson realiza la siguiente tipología de los contextos de aprendizaje en cuatro tipos: 1) contextos pavlovianos clásicos, de un claro carácter conductista basados en las respuestas al estímulo condicionado mediante conductas; 2) contextos de recompensa instrumental o escape, los cuales están basados en la recompensa a la conducta realizada a partir de algún acto dentro del contexto del repertorio conductual; 3) contextos de evitación instrumental, los cuales también funcionan a partir del repertorio conductual y se establecen como parte de un experimento, que incluye cierto tipo de castigo, por ejemplo, descargas eléctricas; 4) contextos del aprendizaje serial y memorístico, en los cuales se desarrollan respuestas del sujeto como muestra de lo aprendido, es decir, por el estímulo condicionado predominante en tanto los actos son realizados.
} 
[Author pre-print: Accepted versión] Citation: Luján, Juan David (2019). Complejidad en clave docente. En Sánchez, Amaya, Tomás y Durango Lara, Ibeth (comps.), Encuentro de socialización de experiencias investigativas en la Facultad de Ciencias y Educación. Memorias, número 5 (pp. 267-279). Bogotá: Universidad Distrital Francisco José de Caldas.

contextos de aprendizaje resalta la complejidad psicológica del sujeto y el reconocimiento de la forma en la cual puntúan o se clasifican los eventos y las circunstancias. A partir de los resultados se direccionan las respuestas para formar y mantener el carácter de quien aprende.

Una lección batesionana más. En su análisis de quién aprende a disparar un rifle, Bateson ([1979] 1993, p. 216) nos dice que esta operación incluye secuencias cibernéticas autocorrectivas, lo que significa incluir contextos de aprendizaje (las secuencias), las acciones de control regulatorias del sistema mental y corpóreo (lo cibernético) y la información que será utilizada (la autocorrección) para lograr la aprehensión de los datos por parte del sistema. Este es un problema que se establece sobre la habilidad del que aprende a tirar para delimitar una diferencia entre la dirección en la cual apunta el cañón y dónde se encuentra el blanco al que se desea atinar al dirigir la mira, frente a la capacidad de su alineación y la vista que el individuo posee.

La idea de Bateson implica concebir la información en un nivel o proceso no-verbal, kinésico, el cual dinamiza la autocorrección. Las experiencias previas y las habilidades acumulativas plantean concebir el aprendizaje como un proceso discontinuo, no-lineal desde la teoría del caos: "no puede concebirse un mundo de los sentidos, de la organización y la comunicación, sin discontinuidad, sin umbrales” (Bateson, [1979] 1993).

¿Es relativista esta posición? La configuración que plantea Bateson sobre calibración y retroalimentación en tanto niveles de control de los sistemas vivientes se clarifica en el intento de jerarquización o tipificación lógica emprendido en su esfuerzo de encontrar la pauta que conecta, la relación, el vínculo.

Más que un relativismo, esta posición es de demarcación; lo que nos atrevemos a decir esclarece algo que luego se vuelve a tornar gris — solía decir Bateson—. "El pensamiento 
[Author pre-print: Accepted versión] Citation: Luján, Juan David (2019). Complejidad en clave docente. En Sánchez, Amaya, Tomás y Durango Lara, Ibeth (comps.), Encuentro de socialización de experiencias investigativas en la Facultad de Ciencias y Educación. Memorias, número 5 (pp. 267-279). Bogotá: Universidad Distrital Francisco José de Caldas.

crece del pensamiento" y más que la naturaleza de los datos lo que importa es reflexionar sobre ellos, lo que nos lleva al siguiente paso (Mead, 1977, p. 171). Con gran atino escribe Bateson ([1979] 1993, p. 219): "El claroscuro está muy bien, pero William Blake nos asegura rotundamente que los hombres sabios ven contornos y por consiguiente los trazan”.

\section{La complejidad se basa en el trabajo por problemas [T1]}

Desde las CC algunos autores como Maldonado (2015) plantean la necesidad de comprender que los aspectos que encierran el problema de la complejidad van mucho más allá de la jerga habitual gestada en torno a los conceptos de complejidad, no-linealidad, auto-organización, criticalidad auto-organizada, emergencia, interacción, sincronización, entre otros.

Como se recuerda, las CC se basan en el trabajo por problemas, más allá de poner su foco en temas, campos disciplinares, objetos de estudio o áreas. Dentro de las denominadas CC los principales complejólogos desarrollan ideas y formas de trabajo alrededor de la definición de problemas $\mathrm{P}$ versus NP. Los primeros se centran en precisarlos como susceptibles de tratabilidad, es decir, su resolución se puede concebir de manera razonable en su tiempo de ejecución algorítmica mediante elementos que permitan su abordaje en un tiempo polinómico.

Los segundos, denominados también como problemas NP-duros o NP-completos (es decir intratables) (Brandes y Thomas, 2005), establecen un nivel complicación mayor; su resolución, aunque puede resolverse en un tiempo polinómico, radica en comprobar si algo puede ser la solución de ese problema.

Dado que $\mathrm{P}$ está en NP y constituyen un súperconjunto (ya que incluye a todos los elementos, tanto a P como a NP), el Clay Mathematics Institute paga un millón de dólares a 
[Author pre-print: Accepted versión] Citation: Luján, Juan David (2019). Complejidad en clave docente. En Sánchez, Amaya, Tomás y Durango Lara, Ibeth (comps.), Encuentro de socialización de experiencias investigativas en la Facultad de Ciencias y Educación. Memorias, número 5 (pp. 267-279). Bogotá: Universidad Distrital Francisco José de Caldas.

quien demuestre la resolución del problema denominado P=NP. Si un problema $\mathrm{P}$ está incluido en NP, la pregunta que intentan responder de modo matemático es la siguiente: ¿Si alguien encuentra que un problema que sea NP se puede demostrar que no es P? A quien responda la cuestión se le ofrece una paga de un millón de dólares. Si lo sabe puede cobrar su primer millón de dólares".

Esta discusión pertenece a la complejidad computacional; actualmente existen alternativas a la resolución de problemas difíciles mediante algoritmos, los cuales también se los entiende como problemas de optimización (o mejora), por lo general tratados a través de metaheurísticas e ideas similares derivadas de metáforas evolutivas. Es de notar que estos problemas constituyen diferentes tipos de complejidad y los algoritmos o formas de resolverlos también tienen su propia complejidad frente a la del problema. "El problema de $\mathrm{P}$ versus NP es determinar si cada lenguaje aceptado por un algoritmo no determinístico en tiempo polinomial también es aceptado por algún algoritmo (determinista) en tiempo polinomial" (Cook, 2006, p. 87).

Se dice que el tiempo polinómico ${ }^{3}$ significa que el tiempo de realización de un algoritmo (en la búsqueda de solución al problema planteado) es menor que un cierto valor calculado a partir del número de variables implicadas en el problema. Esto quiere decir que el tiempo polinomial es diferente a una distribución exponencial, dado que el crecimiento de este último puede ser inimaginable en su tratamiento computacional. A este nivel no existe ordenador capaz de computar un nivel exponencial semejante para este tipo de problemas. El nivel de estos problemas, a los cuales Bateson llamaba decisivos, nos remite a las implicaciones epistemológicas de lo que él objetó: ¿Cómo nosotros podemos saber alguna $\cos a ?$ ¿Cómo un organismo vivo puede subsumir algún tipo de conocimiento aglutinante?

\footnotetext{
${ }^{3}$ Un polinomio es una suma finita de productos entre variables, o de modo sencillo, es la suma de monomios.
} 
[Author pre-print: Accepted versión] Citation: Luján, Juan David (2019). Complejidad en clave docente. En Sánchez, Amaya, Tomás y Durango Lara, Ibeth (comps.), Encuentro de socialización de experiencias investigativas en la Facultad de Ciencias y Educación. Memorias, número 5 (pp. 267-279). Bogotá: Universidad Distrital Francisco José de Caldas.

Este es un problema difícil e incluye lo que podemos llamar mente, y su gran enigma, la conciencia.

\section{Humanidades digitales [T1]}

Las humanidades digitales (HD) o informática humanística — como se le conocía hasta hace algún tiempo-, son una perspectiva que se basa en la aplicación de estilos investigativos a una serie variada de objetos y sujetos de investigación. Más que una definición del campo y sus disputas sobre lo que es y no es, y su continua construcción (Ramsay 2011), las HD se desarrollan con base en referencias a la investigación, la enseñanza y la innovación tecnológica entre la intersección de las humanidades y la computación.

A raíz de esto, su importancia creciente radica en la fertilidad de visualizaciones creativas, representaciones geoespaciales, espacios simulados y análisis de redes de sistemas complejos. Este espacio heterogéneo por lo general se constituye al margen de las instituciones educativas y es percibido bajo una clasificación habitual de interdisciplinariedad.

En cuanto a los niveles disciplinares o corrientes, Gibbs (2011, p. 290) propuso algunas temáticas, campos y perspectivas de las HD a partir de su búsqueda en la comunidad Wiki; sus resultados fueron los siguientes:

[Inicio de cita] Variación de la aplicación de la tecnología al trabajo de humanidades [...] trabajar con medios digitales o un entorno digital [...] minimizar la diferencia entre DH y humanidades [...] naturaleza paraguas o manta de la etiqueta $\mathrm{DH}$; temas a los que los humanistas observan [...] utilizar lo digital y el estudio digital [...] negativas a 
[Author pre-print: Accepted versión] Citation: Luján, Juan David (2019). Complejidad en clave docente. En Sánchez, Amaya, Tomás y Durango Lara, Ibeth (comps.), Encuentro de socialización de experiencias investigativas en la Facultad de Ciencias y Educación. Memorias, número 5 (pp. 267-279). Bogotá: Universidad Distrital Francisco José de Caldas. definir el término [...] método y comunidad [...] digitalización/archivos (y) estudio de lo tecnología digital. [Fin de cita]

En Latinoamérica existen diferentes asociaciones de $\mathrm{HD}^{4}$. Terras et al. (2013) realizaron una compilación en la que intentaron articular de manera crítica las distintas perspectivas en las cuales estas se desarrollan. Recuerdan que el campo se denomina de esta manera a partir del 2005, con la publicación de la editorial Blackwell de su Companion to Digital Humanities, aunque reconocen que desde 1966 esta idea se gestó en el primer journal dedicado a esta materia: Computers and the Humanities, publicado por la editorial Springer.

El vínculo entre HD y CC es tímido. Al situar las CC dentro de las perspectivas de las HD figuran dos tendencias investigativas de vanguardia: las redes sociales (RS) y el campo de los sistemas complejos adaptivos (SCA). En el primer caso, las RS figuran en algunos índices con relación a las humanidades digitales y en menor medida se localizan los SCA. Una búsqueda rápida en los libros de Google nos dice que el campo de las HD desde 1996 hasta 2008 recién estaba ganando terreno en comparación con los SCA y las RS como campos de investigación posicionados (figura 1).

Figura 1. Búsqueda de los terminos complex systems, social networks y digital humanities en los libros de Google entre 1966 a 2008. La figura muestra la baja relacion entre sí de estos tres tópicos

\footnotetext{
${ }^{4}$ Algunos espacios donde se desarrolla esta corriente son: en Colombia, la Red Colombiana de Humanidades Digitales http://rehdi.co/; en Argentina, la Asociación de Humanidades Digitales http://aahd.com.ar/quienessomos; en México, el grupo RedHD http://www.humanidadesdigitales.net/; en República Dominicana, el Observatorio de las Humanidades Digitales https://yellow.place/es/observatorio-humanidades-digitales-santodomingo-dominican y en España, la Asociación Humanidades Digitales Hispánicas http://www.humanidadesdigitales.org/inicio.htm;jsessionid=286A335DEABAD7947CAB254E0A9925AC
} 
[Author pre-print: Accepted versión] Citation: Luján, Juan David (2019). Complejidad en clave docente. En Sánchez, Amaya, Tomás y Durango Lara, Ibeth (comps.), Encuentro de socialización de experiencias investigativas en la Facultad de Ciencias y Educación. Memorias, número 5 (pp. 267-279). Bogotá: Universidad Distrital Francisco José de Caldas.
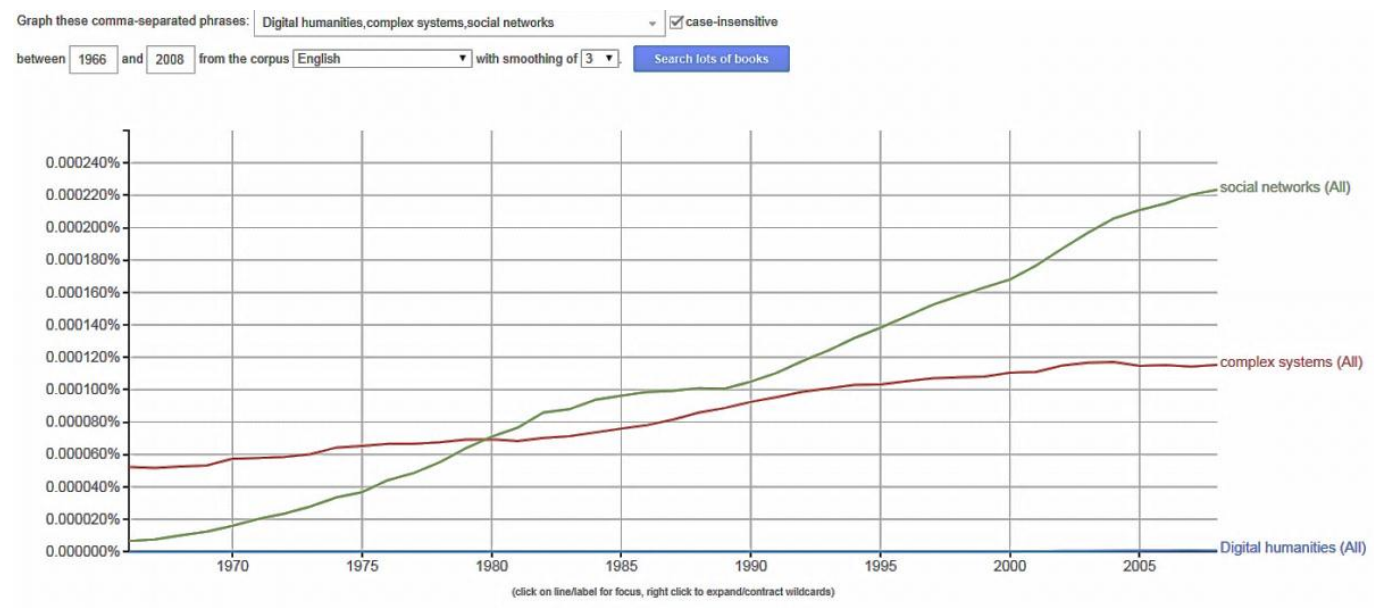

Figura 2. Búsqueda del término digital humanities y Digital Humanities en los libros de

Google. En la gráfica se observa que solo hasta 1993 el vocablo aparece en la literatura
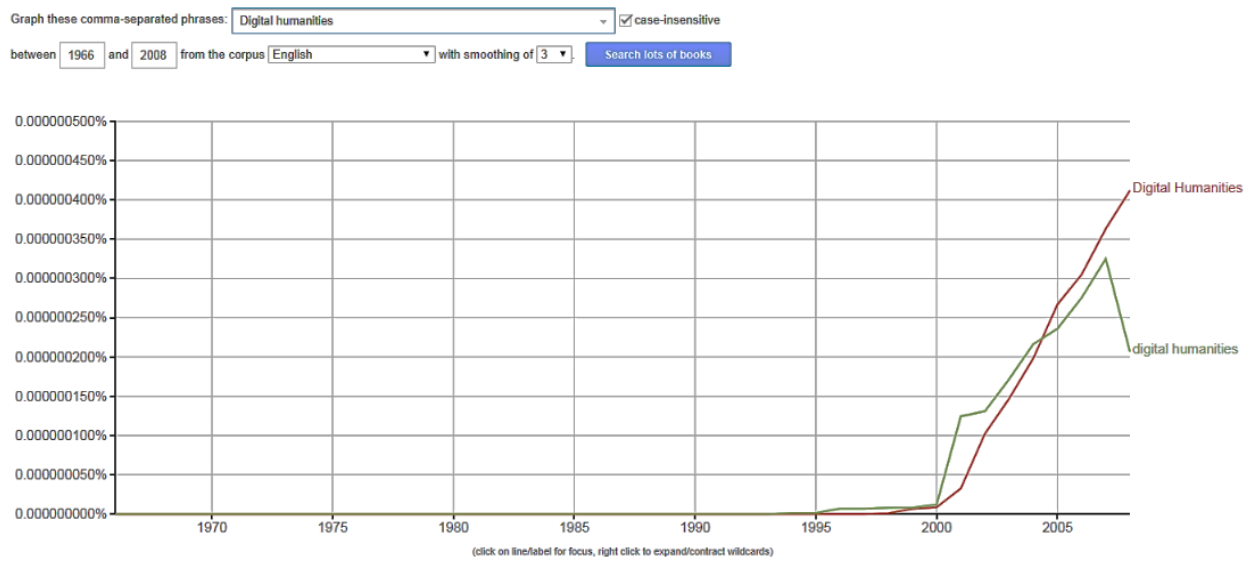

Desde 1993 inicia el apogeo de las HD. A fin de presentar de manera práctica la relación que pretendemos establecer entre algunos modelos de trabajo de las $\mathrm{HD}$, su riqueza técnica y la articulación de problemas complejos, haremos la siguiente contextualización con algunos ejemplos de lo que estas pueden realizar. Veamos un ejemplo de trabajo con un software disponible de HD que permite ejecutar un análisis de texto básico. La plataforma 
[Author pre-print: Accepted versión] Citation: Luján, Juan David (2019). Complejidad en clave docente. En Sánchez, Amaya, Tomás y Durango Lara, Ibeth (comps.), Encuentro de socialización de experiencias investigativas en la Facultad de Ciencias y Educación. Memorias, número 5 (pp. 267-279). Bogotá: Universidad Distrital Francisco José de Caldas.

es Voyant Tools, donde simplemente se introduce el texto que se desea inquirir y su información analítica textual se revela.

Figura 3. Imagen de análisis de un texto digital (ejecutado por el autor con Voyant Tools) ${ }^{5}$

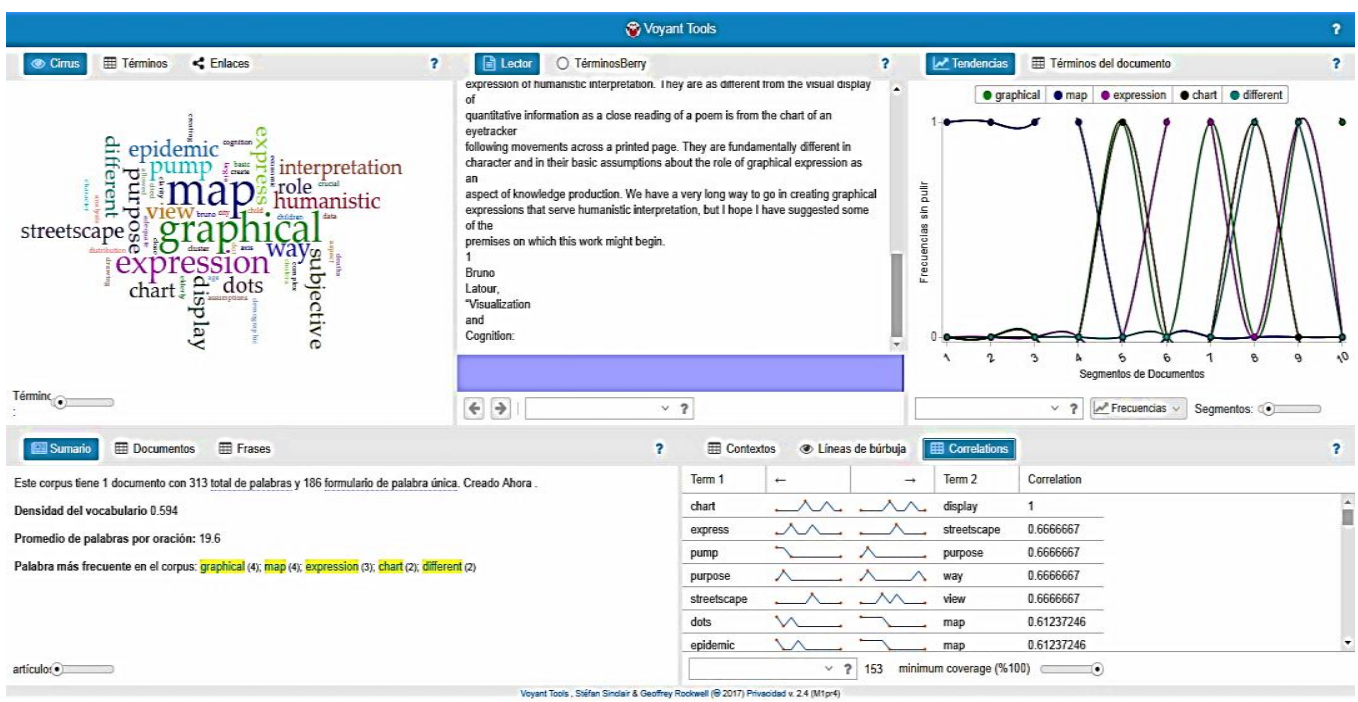

La figura 3 presenta la plataforma Voyant Tools realizando un análisis desde una perspectiva textual cualquiera. Los datos se puede plotear y exportar para una posterior interpretación. Se advierte que este tipo de trabajos requieren para el estudioso armarse de una gran paciencia, ya que el procesamiento no es inmediato y menos la comprensión de lo que se está infiriendo. Como resultado, esta labor demanda diferentes tiempos, los cuales pueden variar dependiendo de lo que se busque.

De nuevo volvemos a la importancia de la definición sobre lo que consideramos un problema más allá de lo que implica la pregunta abordada. Más significativo aún, las implicaciones de lo que definamos como modelo investigativo conciernen de manera directa en lo que establezcamos como problema. Aspectos como la densidad del 
[Author pre-print: Accepted versión] Citation: Luján, Juan David (2019). Complejidad en clave docente. En Sánchez, Amaya, Tomás y Durango Lara, Ibeth (comps.), Encuentro de socialización de experiencias investigativas en la Facultad de Ciencias y Educación. Memorias, número 5 (pp. 267-279). Bogotá: Universidad Distrital Francisco José de Caldas.

vocabulario, el promedio de palabras por oración, los términos del documento, el número de correlaciones entre palabras y su porcentaje, las frecuencias y los segmentos de los documentos, son susceptibles de crear un cuadro comparativo interesante; pueden vislumbrarse además patrones que de otra manera serían imposibles de reconocer. Todo depende del tipo de problema al que nos enfrentemos y el modelo aplicado para su encuadre investigativo.

Para observar las posibilidades de esta aplicación, Voyant Tools también ofrece la visualización de cualquier producción textual mediante gráficos de dispersión.

Figura 4. Imagen de las páginas de este documento (realizado por el autor con Voyant

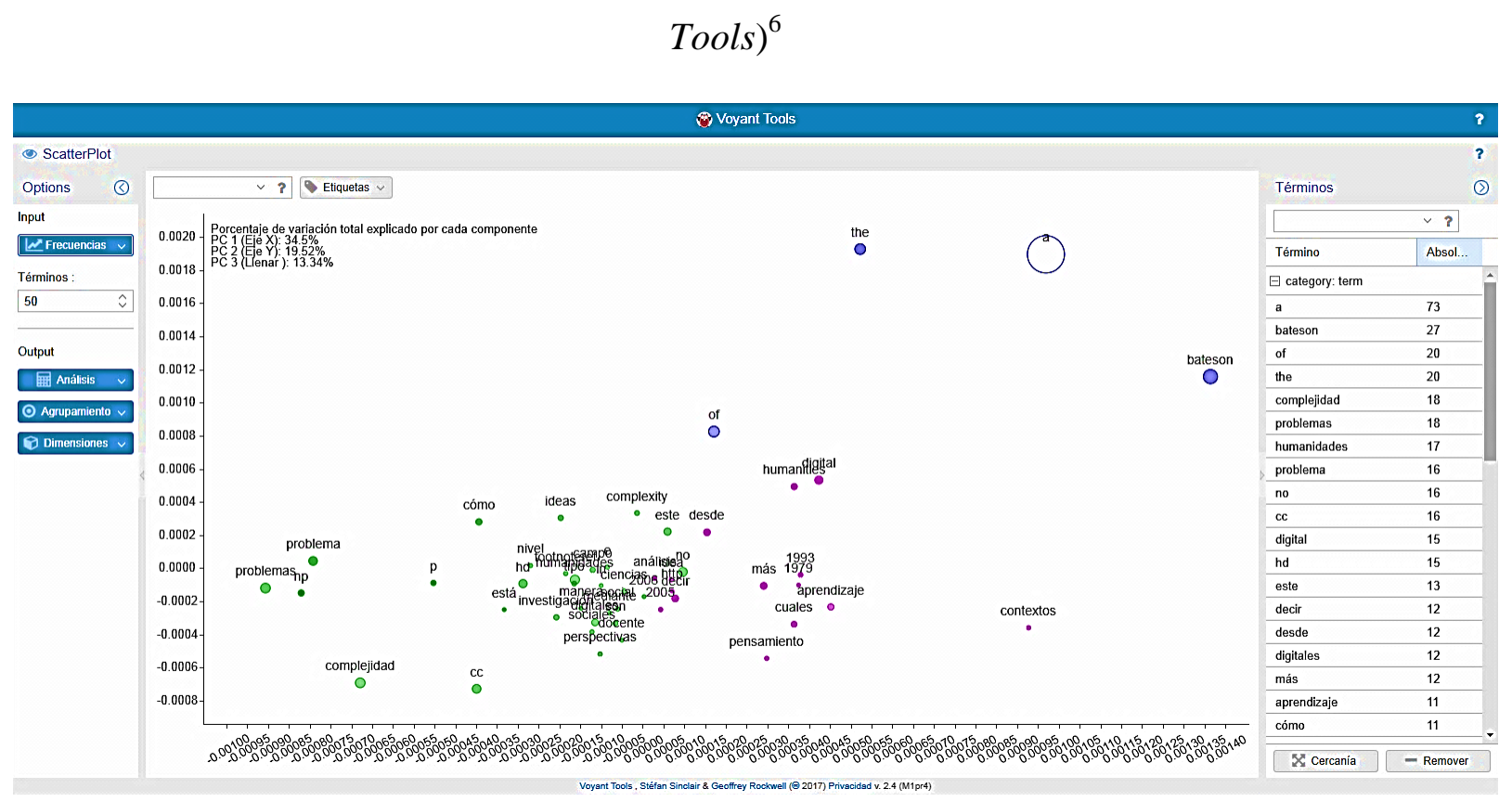

En la figura 4 se analizó el documento que se está leyendo, nótese cómo las palabras Bateson, complejidad, problemas, humanidades, entre otras, figuran como centrales dentro

\footnotetext{
${ }^{6}$ http://voyant-tools.org/?view=scatterplot
} 
[Author pre-print: Accepted versión] Citation: Luján, Juan David (2019). Complejidad en clave docente. En Sánchez, Amaya, Tomás y Durango Lara, Ibeth (comps.), Encuentro de socialización de experiencias investigativas en la Facultad de Ciencias y Educación. Memorias, número 5 (pp. 267-279). Bogotá: Universidad Distrital Francisco José de Caldas.

de esta comunicación. Además esta gráfica presenta el porcentaje de la variación total explicada por cada componente y la técnica de análisis por componentes principales, con un agrupamiento de tres sobre tres dimensiones utilizando frecuencias relativas.

En la figura 5 se muestra otro ejemplo de lo que las HD pueden ofrecer. A través de una sencilla tarea ejecutada en la aplicación Netlytic —que funciona online—, se realizó un análisis de la red social de Facebook, de una librería y sus post. Se examinaron 100 publicaciones puestas en la cuenta, dando como resultado una red de 50 nodos y 204 vínculos.

Un análisis textual realizado sobre esta base de datos al seleccionar la categoría de nombres arrojó 72 resultados; sobre estos se establecieron cadenas de palabras comprendidas durante abril a mayo. Además, la aplicación nos permitió visualizar otras prestaciones estadísticas, informáticas y de análisis comparativo.

Figura 5. Análisis textual realizado por el autor con Netlytic ${ }^{7}$

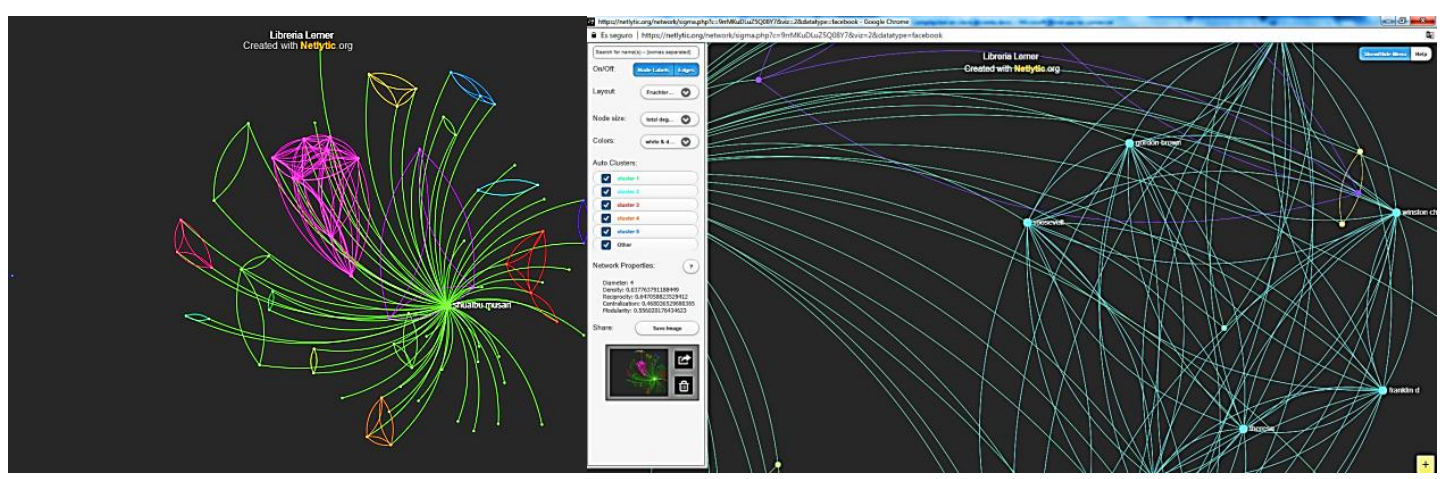

\footnotetext{
${ }^{7}$ https://netlytic.org/home/
} 
[Author pre-print: Accepted versión] Citation: Luján, Juan David (2019). Complejidad en clave docente. En Sánchez, Amaya, Tomás y Durango Lara, Ibeth (comps.), Encuentro de socialización de experiencias investigativas en la Facultad de Ciencias y Educación. Memorias, número 5 (pp. 267-279). Bogotá: Universidad Distrital Francisco José de Caldas.

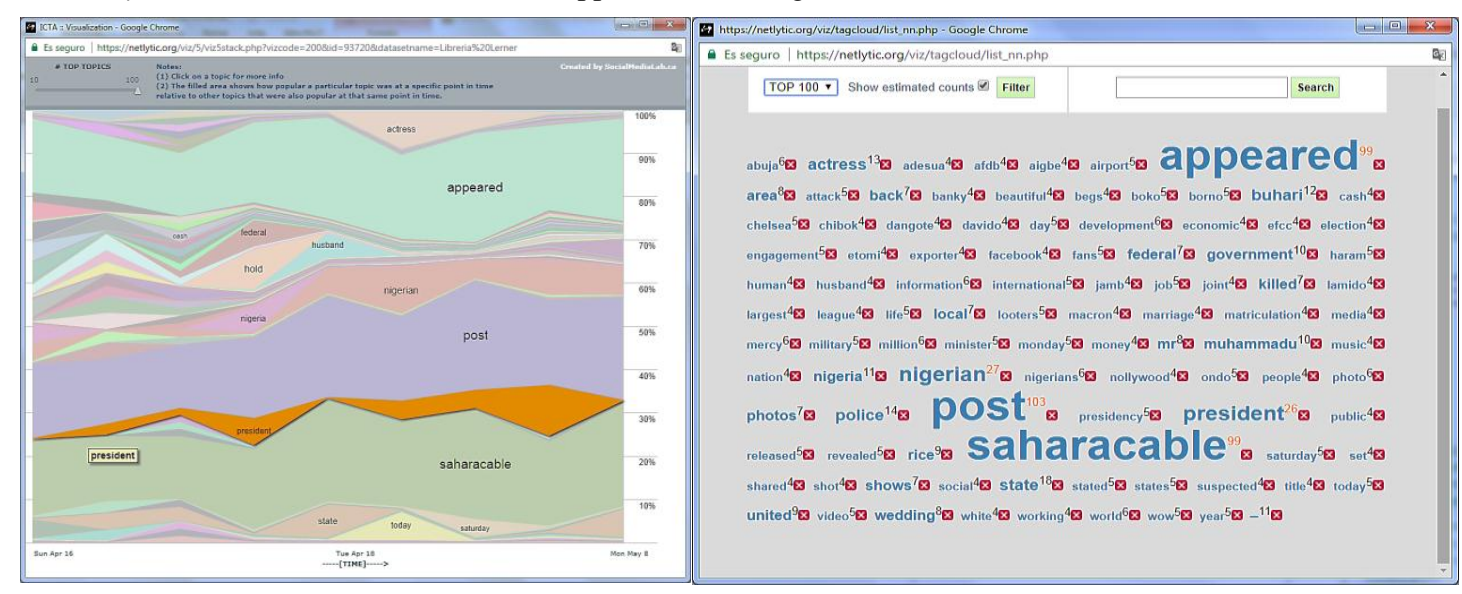

En la parte de arriba se muestra la red de nombres encontrados en los post, mientras que abajo se presenta la visualización de los términos y sus porcentajes, la nube de palabras y su número de apariciones.

Un último ejemplo ofrecido a los lectores es la aplicación Imageplot, la cual es una macro que se ejecuta con el conocido programa ImageJ $J^{8}$. Este programa fue diseñado para el análisis de colecciones visuales y audiovisuales, con el objetivo de abordar las imágenes como documentos y conocer sus características formales. La aplicación visualiza el conjunto en un amplio grupo de datos que pueden ser escalados en orden, tamaño, valores y demás características visuales. En los materiales audiovisuales este programa puede establecer patrones sobre el tiempo y otros aspectos susceptibles de comparación, además de posibilitar animaciones de los corpus visuales analizados.

\footnotetext{
${ }^{8} \mathrm{https}: / /$ imagej.net/Welcome
} 
[Author pre-print: Accepted versión] Citation: Luján, Juan David (2019). Complejidad en clave docente. En Sánchez, Amaya, Tomás y Durango Lara, Ibeth (comps.), Encuentro de socialización de experiencias investigativas en la Facultad de Ciencias y Educación. Memorias, número 5 (pp. 267-279). Bogotá: Universidad Distrital Francisco José de Caldas.

Figura 6. Imagen de los metadatos de entrada analizados de las pinturas de Vincent van

Gogh a partir de sus características (generado por el autor con Imageplot 2014 y sus bases

$$
\text { de datos })^{9}
$$

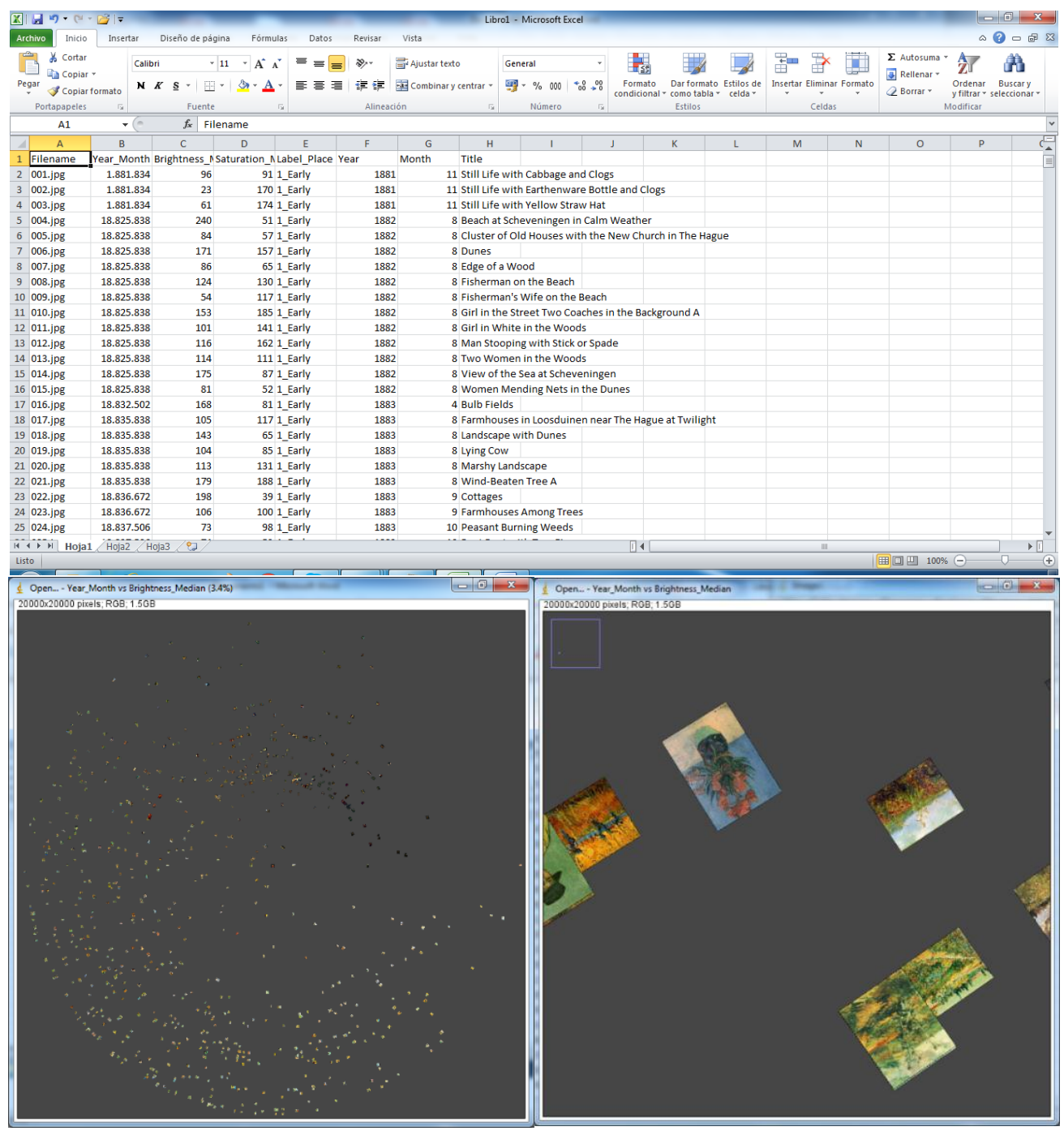

\section{Conclusiones [T1]}

En este escrito intentamos definir una línea de trabajo concreta a partir de las CC y sus aportes al inmenso campo de la labor docente a un nivel investigativo. La idea de comprender la naturaleza de las cosas y el sentido de la mente humana es una ruta que

\footnotetext{
${ }^{9}$ http://lab.softwarestudies.com/p/imageplot.html
} 
[Author pre-print: Accepted versión] Citation: Luján, Juan David (2019). Complejidad en clave docente. En Sánchez, Amaya, Tomás y Durango Lara, Ibeth (comps.), Encuentro de socialización de experiencias investigativas en la Facultad de Ciencias y Educación. Memorias, número 5 (pp. 267-279). Bogotá: Universidad Distrital Francisco José de Caldas.

inició Bateson en muchos aspectos. Hoy en día sus aportes son valorados en distintos campos de trabajo científico, por eso la necesidad de articular a través de sus ideas las perspectivas de la base de las CC y nuestro aporte al insinuar a las HD como una de sus posibles vertientes.

La articulación que se pretende realizar entre las CC, los problemas, el aprendizaje y las humanidades digitales como un campo emergente eficaz en la producción de conocimiento, tiene la intención de visualizar un posible espacio de trabajo con un claro estilo transdisciplinar. Intentamos establecer una simetría entre aspectos que aparecen desconectados en apariencia, pero al mirar con detenimiento se encuentran "relaciones similares entre las partes" (Bateson, [1979] 1993, p. 19).

La semejanza propuesta pretende continuar por una vía que sabemos hoy es transitable. La computación depende en gran medida de las humanidades a propósito de la elaboración del pensamiento y el compromiso académico. Más allá de proponer una lista enorme de incertidumbres intentamos establecer conexiones mediante pautas no solo disciplinares, sino a partir de las múltiples relaciones de distintos órdenes. El docente actual tiene la responsabilidad de estar a la altura de los retos que se avecinan con los cambios económicos, sociales y políticos latinoamericanos.

Los problemas actuales nos obligan a imaginar una pauta propia de lo que inquirimos. Las relaciones con las que establecemos el pensamiento nos invitan a pensar precisamente en las formas en que - en un lenguaje batesoniano- se crean pautas. Constituir las proporciones de esas pautas podría ser el primer problema. El docente se enfrenta al pensamiento de nativos tecnológicos, los cuales no solo tienen pre-saberes, también los modelan. Sabemos que los usos tecnológicos desbordan nuestro propio pasado: ¿son los usos tecnológicos relevantes independientemente de nuestra formación? 
[Author pre-print: Accepted versión] Citation: Luján, Juan David (2019). Complejidad en clave docente. En Sánchez, Amaya, Tomás y Durango Lara, Ibeth (comps.), Encuentro de socialización de experiencias investigativas en la Facultad de Ciencias y Educación. Memorias, número 5 (pp. 267-279). Bogotá: Universidad Distrital Francisco José de Caldas.

$\mathrm{Si}$ la tecnología se convierte en acciones, estas delimitan el contexto y la relevancia de lo que investigamos. La pregunta genera el contexto decisivo de lo que examinamos, ya vimos la importancia de este tipo de razonamientos, aunque siempre habrá lugar para considerar lo que comprendemos por significado, "el significado de lo investigado".

\section{Referencias bibliográficas [T1]}

Bateson, G. ([1971] 1991). Pasos hacia una ecología de la mente. Buenos Aires: Ediciones Planeta-Carlos Lohlé.

Bateson, G. ([1979] 1993). Espíritu y Naturaleza. Buenos Aires: Amorrortu Editores.

Brandes, U. y Thomas E. (comp.). (2005). Network analysis: Methodological foundations. Berlín: Springer Verlag.

Charlton, N. G. (2008). Understanding Gregory Bateson: mind, beauty, and the sacred earth. Albany: State University of New York Press.

Cook, S. (2006). The P versus NP Problem. En J. Carlson, A. Jaffe \& A. Wiles (eds.). (2006). The Millenium Prize Problems. Providence, RI: American Mathematical Society.

Gibbs, F. W. (2011). Digital humanities definitions by type. En M. Terras, J. Nyhan \& E. Vanhoutte (eds.). (2013). Defining Digital Humanities: A Reader (pp. 289-297). London: Ashgate.

Lipset, D. (1980). Gregory Bateson: Legacy of a Scientist. Englewood Cliffs, N.J.: Prentice-Hall.

Longley Arthur, P. \& Bode K. (2014). Advancing Digital Humanities. Research, Methods, Theories. Hampshire, UK: Palgrave Macmillan. https://doi.org/10.1057/9781137337016 
[Author pre-print: Accepted versión] Citation: Luján, Juan David (2019). Complejidad en clave docente. En Sánchez, Amaya, Tomás y Durango Lara, Ibeth (comps.), Encuentro de socialización de experiencias investigativas en la Facultad de Ciencias y Educación. Memorias, número 5 (pp. 267-279). Bogotá: Universidad Distrital Francisco José de Caldas.

Luján, J. D. (2016). Formas de producción sociocultural de la población afrojuvenil en la ciudad de Cali: el caso del rap caleño en la década del noventa (tesis de maestría), Bogotá, Universidad Distrital Francisco José de Caldas.

Maldonado, C. E. (2015). Ciencias de la complejidad, educación, investigación. Tres problemas fundamentales. En C. Correa de Molina, A. Silvera Sarmiento y C. Collante Caiafa. (eds.). Simposio Internacional Educación, Formación Docente y Práctica Pedagógica en Contexto (pp. 49-64). Barranquilla, Universidad Simón Bolívar.

Mead, M. (1977). End linkage: A tool for cross-cultural analysis. En Brockman, J. (ed.). (1977). About Bateson (pp. 169-231). New York: E. P. Dutton.

Morin, E. (2005). Introduction à la pensée complexe. París: Seuil.

Ramsay, S. (2011). On Building. Recuperado de http://stephenramsay.us/text/2011/01/11/on-building/

Reynoso, C. (2016). La prioridad hermenéutica de la pregunta y la prisión del lenguaje. En L. Rodríguez. (coord.). (2006). La emergencia de los enfoques de complejidad en América Latina. Desafíos, contribuciones y compromisos para abordar los problemas complejos del siglo XXI. Tomo I (pp. 99-124). Buenos Aires: Comunidad Editora Latinoamericana.

Terras, M., J. Nyhan, y E. Vanhoutte. (eds.). (2013). Defining Digital Humanities: A Reader. London: Ashgate.

Wallerstein, I. (2005). Las incertidumbres del saber. Barcelona: Gedisa. 